\title{
Responsabilidad Social Empresarial en medios impresos en los departamentos del Atlántico y Magdalena (Colombia)
}

\section{Corporate Social Responsibility in print media in the Atlántico and Magdalena departments (Colombia)}

DOI: https://doi.org/10.17981/econcuc.41.1.2020.Org.3

Artículo de investigación. Fecha de recepción: 06/04/2019 Fecha de devolución: 17/10/2019

Fecha de aceptación: 30/10/2019

Fecha de publicación: 05/11/2019

\section{Camilo Barragán Morales iD \\ Universidad Simón Bolívar. \\ Barranquilla (Colombia) \\ cbarraganm3@unisimonbolivar.edu.co}

\section{Jesús García-Guiliany \\ Universidad Simón Bolívar. \\ Barranquilla (Colombia) \\ jesus.garcia@unisimonbolivar.edu.co}

\author{
Víctor Meza-Rodríguez \\ Universidad Simón Bolívar. \\ Barranquilla (Colombia) \\ vmeza4@unisimonbolivar.edu.co
}

\section{María Mercado Zapata \\ Universidad de la Costa. \\ Barranquilla (Colombia) \\ mafemercado08@gmail.com}

\section{Lucy Olarte Durán Universidad Simón Bolívar. Barranquilla (Colombia) lucyolarte3@gmail.com}

Para citar este artículo:

Barragán, C., García-Guiliany, J., Meza-Rodríguez, V., Mercado, M. \& Olarte, L. (2020). Responsabilidad Social Empresarial en medios impresos en los departamentos del Atlántico y Magdalena (Colombia). Económicas CUC, 41(1), 187-202. DOI: https://doi. org/10.17981/econcuc.41.1.2020.Org.3

\begin{abstract}
Resumen
El artículo tuvo como propósito analizar las empresas de medios de comunicación impresos en los departamentos de Atlántico y Magdalena (Colombia) y cómo estas apropian la Responsabilidad Social Empresarial (RSA) dentro de sus estructuras, teniendo como principal premisa su relacionamiento e impacto con sus grupos de interés, principalmente la comunidad en general. Se desarrolló un enfoque cuantitativo-analítico, donde se utilizó el cuestionario como instrumento para la recolección de los datos, y a su vez, se utilizó el Paquete Estadístico para Ciencias Sociales (SPSS), en su versión 19, como herramienta para el análisis de los datos y posterior presentación de los resultados. Dichos resultados denotan un alto compromiso de parte de estas organizaciones a la hora de instaurar políticas que fomenten la responsabilidad social empresarial, tomando así en consideración los efectos de su accionar para con sus grupos de interés.
\end{abstract}

Palabras clave: Medios de comunicación; medios impresos; responsabilidad social empresarial

\section{Abstract}

The purpose of the article was to analyze the print media companies in the departments of Atlántico and Magdalena (Colombia) and how they appropriate Corporate Social Responsibility (CSR) within their structures, having as main premise their relationship and impact with their stakeholders, mainly the community at large. A quantitative-analytical approach was developed, using the questionnaire as a data collection instrument, and the Statistical Package for the Social Sciences (SPSS), version 19, was used as a tool for data analysis and subsequent presentation of results. These results indicate a high level of commitment on the part of these organizations to implement policies that promote corporate social responsibility, thus taking into consideration the effects of their actions on their stakeholders.

Keywords: Media; print media; corporate social responsibility 


\section{INTRODUCCIÓN}

Las organizaciones, a lo largo de la historia, deben su accionar al entorno en el cual se desenvuelven, es por esto que, más allá de su actividad económica o comercial, y sin importar si ofrece al entorno, productos, bienes o servicios, debe fundamentar dichas acciones dentro de un marco ético de valores compartidos, a fin de ejercer acciones en función de los intereses, así como las necesidades de los grupos relacionados en el que hacer institucional.

De acuerdo a lo expresado por Klisberg (2003) quién concibe la Responsabilidad Social Empresarial (RSE) como pilar fundamental de las instituciones, por tanto, la inclusión de la RSE dentro de los valores corporativos y, a su vez, en el direccionamiento estratégico de la organización resulta vital (Chumaceiro y Hernández, 2012; Paz, Prieto y García, 2015; Chumaceiro, Hernández y Velazco, 2015), aún más para el objeto de estudio de esta investigación, los medios impresos.

Las organizaciones, sin importar su naturaleza, tamaño, estructura o actividad económica, deben crear vínculos que promuevan el mantenimiento de las condiciones del entorno y del bienestar de los actores, lo cual se enmarca dentro de un actuar socialmente responsable (Yepes, Peña y Sánchez, 2007; Chumaceiro, Chirinos y Reyes, 2016). En otras palabras, las empresas no sólo tienen una función económica, sino una función social y ambiental, la cual nace de la necesidad de equilibrar los intereses de la organización, tanto sociales como institucionales (Hartman \& Werhane, 2013; Chumaceiro et al., 2016).

Para Guédez (2006), la RSE promueve ejercicios autónomos a favor de otros, siendo entonces, la capacidad de aceptar obligaciones derivadas de pertenecer a un grupo, organización comunidad o sociedad; conlleva interés y deseo, e implica la razón y al corazón en coincidencia con un propósito que va más allá del individualismo, abriendo a las organizaciones hacia un concepto de altruismo, el cual tiende a representar de igual manera, beneficios para la organización.

Por consiguiente, Colombia no escapa a esta realidad, pues en los últimos 15 años, las empresas han adquirido un compromiso con la sociedad de la que forma parte, involucrándose en la propia estrategia del negocio como un componente más (Chumaceiro et al., 2016; Paz, Harris y Franco, 2016; Cubillos, Montealegre y Delgado, 2016; García, Durán, Parra y Martínez; 2018). Dentro de los planes estratégicos se incorporan políticas concretas de ámbito interno o externo que tienen por objeto desarrollar proyectos de acción social de la propia empresa en colaboración con entidades sin ánimo de lucro.

De igual manera, en los medios de comunicación impresos, conocidos también como diarios, periódicos o prensa del país, específicamente en los departamentos del Atlántico y Magdalena (Colombia), se ha observado, que están constantemente promoviendo y motivando la RSE representando, así, el compromiso de una organización al invertir socialmente en sus empleados, accionistas, clientes, familias y comunidad en general, y así elevar su calidad de vida, lo cual representa un cambio 
de paradigma, de aceptación y de convencimiento en tanto sus acciones deben estar encaminadas hacia la RSE y el beneficio de todos sus grupos de interés (Orozco y Ferré, 2011; Hernández, Muñoz y Barrios, 2017).

Es por ello, que estas empresas, aprovechando su masiva capacidad de difusión y aceptación, desarrollan de manera continua, productos editoriales alternos al periódico, programas y actividades que vayan en pro del rescate de los valores éticos y morales de los ciudadanos (Sisiruca y Salazar, 2014; Hernández et al., 2017), generando conciencia respecto a la conservación del medio ambiente, además de reforzar los principios, derechos y deberes que tienen como seres humanos (Huerta y García, 2009; Chumaceiro et al., 2015).

En este sentido, de acuerdo a un conjunto de entrevistas informales no estructuradas realizadas a personas que toman decisiones relacionadas con planificación en estas empresas, argumentaron que algunas de estas organizaciones han quedado en viejos paradigmas, funcionando sobre criterios carentes de bases sobre nuevas estrategias planificadas (Villasmil y Crissien, 2015; Paz et al., 2016; Cubillos et al., 2016; Durán, Crissien, Virviescas y García, 2017; García, Durán, Cardeño, Prieto, García y Paz; 2017; Hernández et al., 2017). Con actividades responsables socialmente, sin saber a ciencia cierta qué es lo mejor para ellos, a sabiendas que el entorno organizacional lleva a las empresas a orientar sus estrategias con base a la satisfacción plena de las necesidades, deseos, preferencias de sus consumidores (García, García e Cabello, 2017).

Por otra parte, la evaluación de las estrategias de RSE en Colombia desarrolladas por este grupo de empresas, puede ubicarse dentro del contexto histórico en la cual se desenvuelven, desde comienzos de las guerrillas y el paramilitarismo, pasando por la apertura de sus mercados, y las distintas regulaciones en el ordenamiento jurídico, los cuales han incididos en esta selección estratégica, por tanto, el llamado es a asumir por parte de los directivos empresariales, un compromiso mayor, visto desde las aristas de RSE y productividad (García et al., 2018).

Esta situación, probablemente se deba a la obsolescencia en la aplicación de estrategias gerenciales (Durán et al., 2017; García et al., 2017) que fundamenten la planificación en relación a las actividades de RSE enmarcadas en este sector. Lo cual puede haber traído como consecuencia, una planificación estratégica no acorde con la realidad del entorno gerencial competitivo en los medios de comunicación impresos, por lo que no se ha contemplado de manera efectiva en el entorno interno de las acciones de RSE.

Particularmente, en el caso de los departamentos del Atlántico y Magdalena, las empresas de comunicación masiva, de igual forma que las demás organizaciones del entorno, deben apropiar y asociar a su cultura, la RSE. Incluyendo las políticas, estrategias y acciones que estas prácticas requieren, y de igual manera, y tomando en cuenta su actividad como forma de divulgación a la comunidad, estas políticas, estrategias y acciones pueden resultar con una mayor efectividad, debido esto, por supuesto, a la masividad y el alcance de estas organizaciones. 
De allí, que surja la necesidad de llevar investigaciones que permitan analizar la RSE en medios de comunicación impresos en los departamentos de Atlántico y Magdalena, caracterizando los elementos que la componen, y determinando el compromiso en las prácticas de RSE, suministrando a las empresas estudiadas, la información necesaria para orientar sus estrategias de cara a las nuevas exigencias y tendencias del desarrollo actual, donde las organizaciones cada día buscan estar a la vanguardia, y en condiciones óptimas para ejecutar estas acciones.

\section{Desarrollo}

\section{Responsabilidad Social Empresarial}

La RSE representa una nueva tendencia de cambio en las organizaciones, por cuanto deben ser compatibles con el desarrollo de todo el entorno social, convirtiéndose en verdaderos ejes de crecimiento para la sociedad, dentro de un ámbito de eficiencia que les permita a las organizaciones ser sustentables.

Sin embargo, la RSE no es algo novedoso en el mundo empresarial, aunque en la última década está adquiriendo una nueva dimensión por el especial interés que conlleva, demandando una gestión cuidada como en cualquier ámbito de valor estratégico, que supere lo anecdótico o lo filantrópico (Paz et al., 2015, 2016; García et al., 2018). En este sentido, se observa la existencia de una pluralidad de acciones para un mismo concepto, dado que las organizaciones asumen a su conveniencia y percepción como abordar este tema ante sus grupos de interés.

Olcese, Rodríguez y Alfaro (2008), Chumaceiro et al. (2015) y Paz et al. (2016), declaran que la RSE constituye todas aquellas prácticas que evidencian por parte de las organizaciones, la apertura y sensibilidad con el entorno, además de trabajo mancomunado para la sociedad y construcción de confianza. En este sentido, Melamed-Varela, Blanco-Ariza y Rodríguez-Calderón (2018) mencionan la necesidad de crear un nuevo paradigma empresarial que toma como base, las relaciones sociales y la gestión ambiental, de tal forma que, resulta pertinente considerar las interacciones colaborativas entre todos los actores involucrados dentro de sus hojas de ruta estratégica en pro del desarrollo empresarial y de un ejercicio gerencial efectivo.

Rabasa (2005) considera que, los antecedentes históricos de la RSE se remontan a siglos pasados y se encuentran en los propios orígenes de las organizaciones que conjugan capital y trabajo para generar riqueza y beneficio, aunque en el camino los abusos sobre los trabajos hayan sido notorios. De esta forma, pueden recordarse las condiciones de explotación a las cuales los trabajadores se vieron sometidos en el periodo de la "Revolución Industrial", sin remontarse a épocas más lejanas, en donde la esclavitud fue la base de la economía de las sociedades más modernas.

Para Bateman y Snell (2005), "la empresa socialmente responsable maximiza los efectos positivos sobre la sociedad y minimiza sus efectos negativos" (p. 147), es decir, actividades que generen beneficios constantes para las comunidades que las rodean, mientras que sus acciones a mejorar sean mitigadas. 
Aguilera y Puerto (2012) conciben la RSE como una nueva alternativa para "competir", ya que esta permite transmitir valor agregado a la sociedad. En tal sentido, Chumaceiro et al. (2015) y Guédez (2006) considera que la RSE es la capacidad para comprender los alcances de los compromisos sociales de la empresa, implicando acciones concretas, para crear mejoras en los grupos sociales vinculados a las empresas, tanto en el nivel interno como externo de las mismas. Desde un punto de vista estratégico, se espera por parte de la organización ver estas acciones representadas en ventajas competitivas, siendo tarea de la organización reconocer en que momento estas acciones han rendido estos frutos, ya que a partir de estos esfuerzos colaborativos, se impulsa el crecimiento y sostenibilidad integral de las organizaciones.

Para efectos de este estudio, se considera la RSE desde la perspectiva de Méndez (2005), quién la califica como el conjunto de esfuerzos que realizan las empresas del sector de medios de comunicación impresos, a los fines de integrar sus acciones en la protección de los elementos sociales y ambientales, atendiendo a las preocupaciones sociales y medioambientales que circulan a los medios de comunicación impresos en los departamentos del Atlántico y Magdalena.

Por tanto, deben orientar sus labores misionales con base a la RSE, siendo su principal misión como empresa informativa está marcada por el servicio (de información) a la comunidad local a la que atiende, dado que estos poseen alta circulación y por ende guardan una relación muy cercana con las comunidades, a razón de que sus instalaciones están ubicadas en las zonas de mayor densidad de población de la localidad (Caro, 2006).

\section{Elementos de la Responsabilidad Social}

La RSE cumple integralmente con la finalidad de la empresa en sus dimensiones económicas, sociales y ambientales en sus contextos internos como externos, considerando las expectativas de todos sus participantes.

En este sentido, González (2005) afirma que la RSE conlleva la actuación comprometida de mejora continua, permitiendo a la empresa ser más competitiva, respetando y promoviendo el desarrollo pleno de las personas y de las comunidades en que opera en el entorno, atendido las expectativas de todos sus participantes, como pueden ser: Clientes o consumidores, proveedores, accionistas, autoridades, competidores, personal, directivos, tanto organizacionales, sociales o medioambiente, así como comunidades en que opera (Paz et al., 2016; Cubillos et al., 2016). También hace énfasis en los elementos que determinan la dimensión económica interna de la RSE, indicando que deriva en la generación de un valor agregado entre colaboradores y accionistas, considerando no solo las condiciones de mercado sino también la equidad o justicia.

Por tanto, un Stakeholder, según Weiss (2006), es cualquier individuo o grupo que pueda afectar o sea afectado por las acciones, decisiones, políticas, prácticas o metas de la organización. De igual forma, mencionan los elementos económicos externos, los cuales implican la generación de bienes/servicios útiles para la comunidad, así 
como el apoyo a las causas sociales vía a la contribución impositiva legal, incluyendo las aportaciones para causas sociales seleccionadas (Paz et al., 2016; Cubillos et al., 2016; Chumaceiro et al., 2015).

Por otro lado, en su dimensión social interna, implica el fomento de la calidad de vida en el trabajo y el desarrollo integral de sus trabajadores, la responsabilidad total sobre de las repercusiones sociales y ambientales de sus procesos y sus productos (Chumaceiro et al., 2016; Paz et al., 2015; Durán et al., 2017). Su dimensión social externa, conlleva a la realización de acciones seleccionadas para contribuir a la generación de condiciones que favorezcan el pleno desarrollo de las comunidades además de la preservación de la herencia ecológica común.

En su defecto, las actividades socialmente responsables, como los programas de mejoras sociales, deben ser establecidas por ley, políticas públicas y actividades y contribuciones privadas. De esta manera, los medios de comunicación impresos conciben acciones de RSE, considerando su impacto en el entorno con el cual se vinculan, abarcando tanto el ámbito del personal, sus familiares, así como adjudicando la importancia de las acciones de protección contra la degeneración del ambiente, considerando también por parte de estos, la aplicación asertiva de la RSE y generar contenidos sobre este tema (Ferré y Tolotti, 2010; Chumaceiro y Hernández, 2012).

\section{Sensibilidad Social}

La sensibilidad social representa un punto esencial en el logro de la responsabilidad social efectiva. Moliner (2004) apunta a considerar que el termino sensibilidad social hace referencia a las actividades que van más allá de la obligación y la reacción social. Con base a este criterio, el comportamiento socialmente responsable es anticipadorpreventivo, en lugar de ser reactivo-restaurador. De acuerdo con esta perspectiva, los gerentes progresistas, aplican las destrezas y recursos corporativos a cada problema, como mejorar viviendas, crear compañías pequeñas o empleos para jóvenes, entre otras actividades que busquen el fortalecimiento de estas organizaciones.

Opina Guédez (2006) que los defensores de la sensibilidad social en general defienden este significado de RSE esgrimiendo tres razones, entre ellas las actividades y metas económicas de las empresas que no pueden separarse claramente de las actividades y metas de la sociedad, dado que todas sus decisiones tienen consecuencia sociales. Por tanto, se evidencia que los autores coinciden al considerar la sensibilidad social como un elemento de la responsabilidad social, siendo calificada en términos de la necesidad de participar en las actividades para generar consecuencias favorables a nivel de los problemas sociales evidenciados en los distintos públicos de interés para las empresas.

A los efectos de este trabajo, interesan los planteamientos de Guédez (2006) al considerar la medición de la sensibilidad social en los medios de comunicación impresos, a los fines de responder al objetivo específico de la investigación centrado en caracterizar los elementos de responsabilidad social incluidos en la filosofía de gestión de estas organizaciones. 


\section{Preservación de la Herencia Ecológica}

Actualmente, las preocupaciones ambientales se visualizan con más fuerza y se asumen como una crisis del desarrollo antes que una simple crisis medioambiental (Huerta y García, 2009, Paz et al., 2015; Paz et al., 2016; Cubillos et al., 2016; García et al., 2017). Desde esta perspectiva, Moliner (2004) afirma que las nuevas corrientes de desarrollo relacionadas con el desarrollo sustentable plantean subordinar los objetivos puramente económicos (crecimientos) a objetivos más amplios, que den cuenta ante todo de la vida en sus dimensiones física, psicológica y social.

En este contexto de la herencia ecológica, Guédez (2006) afirma que se debe entender como el conjunto de acciones concertadas entre los diferentes actores en un determinado tiempo y espacio con el propósito de acercarse nuevamente a un equilibrio en las relaciones sociedad-naturaleza. Por tanto, para lograr tales objetivos es esencial la participación de todos los actores, individuales o colectivos, en los procesos de gestión. Por consiguiente, se plantea un enfoque que, en sí mismo no es nuevo, más bien representa la recuperación de cómo abordar la problemática ambiental más allá de plantear respuestas eminentemente técnicas o tecnológicas; lo cual establece un abordaje más integrador, que refleja las relaciones sociedad-naturaleza. Estos planteamientos son relevantes a los fines de medir la orientación de los medios de comunicación impresos, al momento de realizar acciones enfocadas en la responsabilidad social, especialmente en la preservación del medio ecológico; al momento de caracterizar los elementos de responsabilidad social que forman parte de la filosofía de gestión de estas empresas.

\section{Carácter Voluntario}

La RSE, también es definida como el compromiso voluntario de las empresas con el desarrollo de las comunidades y la preservación del medio ambiente desde su composición social (Chumaceiro et al., 2015; Paz et al., 2016; Cubillos et al., 2016; García et al., 2018, García et al, 2017). Moliner (2004) afirma que es un comportamiento responsable hacia las personas y grupos sociales con quienes interactúa, centra su atención en la satisfacción de las necesidades de los grupos de interés a través de determinadas estrategias, cuyos resultados han de ser medidos, verificados y comunicados adecuadamente.

Para Méndez (2005), va más allá del simple cumplimiento de la normativa legal establecida y de la obtención de resultados exclusivamente económicos a corto plazo, supone entonces un planteamiento de tipo estratégica afectando la toma de decisiones.

Guédez (2006) haciendo alusión a las tendencias europeas en materia de responsabilidad social indica que ser socialmente responsable no significa solamente cumplir con las obligaciones jurídicas, sino también ir más allá de sus cumplimientos, invirtiendo más en el capital humano, el entorno y las relaciones con los interlocutores. 
Por tanto, la RSE es considerada a los fines de estos autores con carácter voluntario, es decir, un compromiso voluntario adoptado en la gestión de las organizaciones para actuar en su propio beneficio y en el de sus trabajadores, familia y del entorno social. Entonces, la práctica de RSE implica que las empresas desarrollen una visión integral para el futuro, donde ocurran las mejoras en el entorno de forma inmediata así como los beneficios de la comunidad.

Dicho carácter voluntario para generar bienestar en las comunidades, públicos o personal interno, es importante a nivel del compromiso que demuestran los medios de comunicación impresos en la ejecución de actividades de responsabilidad social, por lo cual es pertinente considerar este aspecto al momento de valorarlo en esta población de interés.

\section{Metodología}

La investigación, estuvo basada en un enfoque cuantitativo, con consideraciones de tipo descriptivas (García et al., 2019; Cazallo et al., 2019) para el análisis de los resultados, en este sentido. Pelekais et al. (2012) describen algunas características fundamentales dentro de un conjunto homogéneo de fenómenos mediante la utilización de criterios sistemáticos permite poner de manifiesto su estructura y comportamiento.

En este caso, caracterizar los elementos de la Responsabilidad Social Empresarial incluidos dentro de la filosofía de gestión de los medios de comunicación impresos y determinar el compromiso de los medios de comunicación impresos en las prácticas de RSE. Lo anterior, mediante un diseño transversal, que se puede aplicar en una sola oportunidad, en un momento único (Hernández, Fernández y Baptista, 2014), no experimental, ya que no se hace cambiar intencionalmente la variable, sino que, por el contrario, observa los fenómenos dados desde su naturaleza, para proceder a analizarlos (Hernández et al., 2014).

De igual manera, la investigación se enmarca bajo la tipología de campo, por tanto, los datos recogidos se toman en forma directa de la realidad, a través de un cuestionario estructurado aplicado a empresas editoriales de los departamentos de Atlántico y Magdalena. Empresas representativas para este caso de la población del estudio; esto refiere a cualquier conjunto de elementos donde se pretenda indagar y conocer algunas o todas sus características, de tal forma, que para la investigación sirvan como insumo para la construcción de resultados, conclusiones y recomendaciones.

En cuanto a la muestra, para efectos de la investigación, se utilizó el método de elección a conveniencia del investigador, destacando entones cinco (5) empresas, tres (3) del departamento de Atlántico y dos (2) del departamento del Magdalena. Dichos cuestionarios fueron analizados por el Paquete Estadístico para Ciencias Sociales (SPSS, según sus siglas en inglés), en su versión 19.

De igual manera, la investigación utilizó media, moda y desviación para establecer la descripción de los resultados. La Tabla 1 muestra los rangos, intervalos que 
lo componen, y según esto a que categoría pertenece cada empresa, en términos de media, moda y desviación estándar.

TABLA 1.

Baremos de Interpretación de la Media, Moda y Desviación Estándar

\begin{tabular}{llllll}
\hline & \multicolumn{2}{l}{ Media y Moda } & \multicolumn{2}{c}{ Desviación Estándar } \\
\hline Rango & Intervalo & Categoría & Rango & Intervalo & Categorías \\
1 & $4.21-5$ & Muy Alto & 1 & $3.21-4$ & Muy Alta Dispersión \\
2 & $3.41-4.20$ & Alto & 2 & $2.41-3.20$ & Alta Dispersión \\
3 & $2.61-3.40$ & Moderado & 3 & $1.61-2.40$ & Moderada Dispersión \\
4 & $1.81-2.60$ & Bajo & 4 & $0.81-1.60$ & Baja Dispersión \\
5 & $1-1.80$ & Muy Bajo & 5 & $0-0.80$ & Muy Baja Dispersión \\
\hline
\end{tabular}

Fuente: Elaboración propia.

\section{Resultados}

Caracterización de los elementos de responsabilidad social incluidos en la filosofía de gestión de los medios de comunicación impresos

A continuación, se representan los hallazgos encontrados en lo referente a los elementos de la RSE incluidos por las empresas objeto de estudio, dentro de su filosofía de gestión (Tabla 2).

TABLA 2.

Indicadores y Dimensión de los elementos de la Responsabilidad Social

\begin{tabular}{lllll}
\hline & \multicolumn{1}{c}{$\begin{array}{c}\text { Sensibilidad } \\
\text { social }\end{array}$} & \multicolumn{1}{c}{$\begin{array}{c}\text { Preservación de la } \\
\text { herencia ecológica }\end{array}$} & $\begin{array}{c}\text { Carácter } \\
\text { voluntario }\end{array}$ & $\begin{array}{c}\text { Elementos de la } \\
\text { responsabilidad social }\end{array}$ \\
\hline $\mathrm{N}$ & 42 & 42 & 42 & 42 \\
Media & 3,71 & 4,07 & 4,14 & 3,88 \\
Moda & 3 & 5 & 5 & 4 \\
Des. Típ. & 1,111 & 1,135 & 1,138 & 1,041 \\
Mínimo & 2 & 2 & 2 & 2 \\
Máximo & 5 & 5 & 5 & 5 \\
\hline
\end{tabular}

Fuente: Elaboración propia.

En la Tabla 2 se muestran los resultados de los indicadores y de la dimensión de los elementos de la Responsabilidad Social. En tal sentido, en el indicador "sensibilización social", se obtuvo que el promedio fue de 3,71; ubicándose así, en el nivel alto. La moda de 3 indica que el valor más frecuente corresponde al nivel alto. La desviación estándar de 1,111 implica baja dispersión de los puntajes alrededor de 
la media, observándose como el valor mínimo presentado por la muestra fue de 2 y el máximo de 5. Indicando que los directivos se muestran altamente interesados y sobretodo, socialmente sensibles ante los problemas comunitarios, indicando que el personal de la empresa se involucra en las actividades de responsabilidad social y se muestra receptivo con los problemas de responsabilidad social.

En referencia al indicador concerniente a la "preservación de la herencia ecológica", se obtuvo que el promedio fue de 4.07, intervalo correspondiente al nivel alto. La moda de 5 refleja que el valor más frecuente corresponde al nivel muy alto. La desviación estándar de 1,135 implica baja dispersión de los puntajes alrededor de la media, observándose como el valor mínimo en la muestra fue de 2 y el máximo de 5. Los resultados denotan altos niveles de ejecución en la preservación ecológica por cuanto se realizan acciones de limpieza, recolección y clasificación de desechos, así como y la conservación de árboles en las áreas adyacentes para contribuir con las condiciones ecológicas.

Investigando el indicador referido al "carácter voluntario", se obtuvo un promedio de 4,14; siendo ubicado en el nivel alto.Lo cual refleja las intenciones del actuar por parte de estas organizaciones. La moda de 5 muestra que el valor más frecuente corresponde al nivel muy alto y la desviación estándar de 1,138 implica baja dispersión de los puntajes alrededor de la media, observándose como el valor mínimo presentado por la muestra fue de 2 y el máximo de 5 .

Estos resultados reflejan la existencia de diversas actividades de responsabilidad social de forma voluntaria, lo cual incluye la participación voluntaria del personal en estas actividades, incentivando a toda la organización y cuentan con planes voluntarios de soporte basados en responsabilidad social para ayudar a las comunidades.

A nivel de la dimensión "elementos de la responsabilidad social", se obtuvo que el promedio fue de 3,88; lo cual le ubica en el nivel alto. La moda con valor 4, significa que el valor más frecuente corresponde al nivel muy alto.

La desviación estándar de 1,041 implica baja dispersión de los puntajes alrededor de la media, observándose como el valor mínimo presentado por la muestra fue de 2 y el máximo de 5.

Estos resultados, conducen a denotar que tales organizaciones están orientadas a fomentar y asumir seriamente sus obligaciones sociales y medioambientales para con la sociedad y con cada uno de los elementos que la integran. Por tanto, y basados en el alcance masivo de este tipo de organizaciones, son las llamadas a abanderar procesos de concientización referentes con la responsabilidad social, comenzando desde su organización. Dentro de estas actividades figura, por ejemplo, el patrocinio a eventos para asistencia médica y deportivos para ciudadanos de bajos recursos, hasta la generación de contenidos que promuevan y fomenten la ejecución de estas acciones por parte de las comunidades en general.

La Tabla 3 representa a manera de indicadores, el compromiso asumido en materia de RSE por las organizaciones. 
TABLA 3.

Indicadores y Dimensión del Compromiso con la Responsabilidad Social

\begin{tabular}{lllll}
\hline & \multicolumn{1}{c}{$\begin{array}{c}\text { Formulación de } \\
\text { Políticas }\end{array}$} & Valores & Fortalecimiento & $\begin{array}{c}\text { Compromiso con la } \\
\text { responsabilidad social }\end{array}$ \\
\hline $\mathrm{N}$ & 42 & 42 & 42 & 42 \\
Media & 4,07 & 4,33 & 4,24 & 4,24 \\
Moda & 5 & 5 & 5 & 5 \\
Des. Típ. & 1,091 & 1,028 & 1,055 &, 958 \\
Mínimo & 2 & 2 & 2 & 2 \\
Máximo & 5 & 5 & 5 & 5 \\
\hline
\end{tabular}

Fuente: Elaboración propia.

En la Tabla 3 se muestran los resultados de los indicadores y de la dimensión "compromiso con la responsabilidad social". En tal sentido, en el indicador "formulación de políticas", se obtuvo que el promedio fue de 4,07; lo cual le ubica en el nivel alto. La moda es 5 denotando que el valor más frecuente corresponde al nivel muy alto. La desviación estándar de 1,091 implica baja dispersión de los puntajes alrededor de la media, observándose como el valor menor presentado por la muestra fue de 2 y el máximo de 5 . Los resultados indican que es alto el nivel de formulación de políticas en materia de responsabilidad social, el sistema de control vela por el cumplimiento de las políticas de responsabilidad social y el personal participa en la ejecución y realización de dichas políticas dentro de los medios de comunicación impresos.

En cuanto al indicador "valores", se obtuvo que el promedio fue de 4,33; lo cual le ubica en el nivel muy alto. La moda es de 5 y refleja que el valor más frecuente corresponde al nivel muy alto. La desviación estándar de 0.958 implica baja dispersión de los puntajes alrededor de la media, observándose como el mínimo presentado por la muestra fue de 2 y el máximo de 5. Es muy alto el nivel en el cual los valores empresariales que incluyen RSE, el sistema de incentivo incluye los valores de responsabilidad social y cuentan con estrategias para fomentar los valores de responsabilidad social en el personal que labora en los medios de comunicación impresos.

En referencia al indicador "fortalecimiento" se obtuvo que el promedio fue de 4,24; lo cual le ubica en el nivel muy alto. La moda de 5 indica que el valor más frecuente corresponde al nivel muy alto. La desviación estándar de 1,111 e implica baja dispersión de los puntajes alrededor de la media, observándose como el valor menor de la muestra fue de 2 y el máximo de 5 . Por tanto, es muy alto el nivel en el cual se considera al fortalecimiento de la responsabilidad social una actividad prioritaria en la empresa, las actividades operativas incluyen la aplicación de acciones para el fortalecimiento de la responsabilidad social y las estrategias de gestión incluyen actividades en esta materia. 
En cuanto a la dimensión "compromiso con la responsabilidad social", se obtuvo que el promedio fue de 4,24 ubicandola en el nivel alto. La moda es 5 y muestra que el valor más frecuente corresponde al nivel alto. La desviación estándar de 0,958 implica baja dispersión de los puntajes alrededor de la media, observándose como el valor mínimo presentado por la muestra fue de 2 y el máximo de 5 . Los resultados indican que es alto el nivel en el cual se produce el compromiso con la responsabilidad social, implicando que tanto el personal gerencial y supervisorio de estas organizaciones, se orienta a realizar expresando la responsabilidad, involucramiento y avocamiento, demostrando compromiso con los públicos de interés de los medios de comunicación impresos.

TABLA 4.

Variable Responsabilidad Social

\begin{tabular}{lll}
\hline \multicolumn{1}{c}{$\mathrm{N}$} & 42 \\
\hline Media & 4,14 & \\
Moda & 5 & \\
Des. Típ. & .926 \\
Mínimo & 2 \\
Máximo & 5 \\
\hline
\end{tabular}

Fuente: Elaboración propia.

En la Tabla 4 se muestran los resultados de la variable Responsabilidad Social. En tal sentido el promedio fue de 4,14, lo cual le ubica en el nivel alto. La moda de 5 indica que el valor más frecuente corresponde al nivel muy alto. La desviación estándar de 0,926 implica baja dispersión de los puntajes alrededor de la media, observándose como el valor mínimo presentado por la muestra fue de 2 y el máximo de 5. Por tanto, se afirma que es alto el nivel de responsabilidad social de los medios de comunicación impresos demostrando grandes competencias para comprender los alcances de los compromisos sociales de la empresa, implicando acciones concretas para crear mejoras en los grupos sociales vinculados a las empresas, tanto en el nivel interno como externo a las mismas.

\section{Conclusiones}

Respondiendo al propósito de la investigación, se identificaron los elementos de responsabilidad social presentes en los medios de comunicación impresos, encontrando que existe un nivel alto de sensibilización social de estas empresas ante los problemas de las comunidades. Así mismo, en referencia a la preservación de la herencia ecológica, se llevan a cabo acciones y estrategias de conservación ecológica, lo cual se realiza con acciones concretas, orientándose a mantener las áreas adyacentes en condiciones óptimas. 
De igual manera, se evidenció el carácter voluntario de las actividades de responsabilidad social, y la participación del personal en los distintos niveles jerárquicos, dando como resultado un cumplimiento satisfactorio, en los elementos de responsabilidad social.

En cuanto al compromiso de los medios de comunicación impresos en las prácticas de la responsabilidad social, se observó el apego en la formulación y cumplimiento de políticas organizacionales. De igual forma, en relación a los valores, se observa muy alto el nivel en el cual se aplican los valores empresariales que incluyen la responsabilidad social, contándose con estrategias para fomentar los mismos. En el mismo orden de ideas, realizan acciones tendentes al fortalecimiento de la responsabilidad social. Resultando por tanto, un nivel alto de compromiso con la responsabilidad social de los medios de comunicación impresos en los Departamentos del Atlántico y Magdalena, denotando competencias para comprender los alcances de los compromisos sociales de la empresa para con sus Stakeholders.

A futuro, se recomienda para estas organizaciones, la articulación de esfuerzos sectoriales para lograr dinamizar las iniciativas de responsabilidad social de cara a sus grupos de interés o Stakeholders.

\section{REFERENCIAS}

Aguilera, A. y Puerto, D. (2012). Crecimiento empresarial basado en la Responsabilidad Social. Pensamiento \& Gestión, (32), 1-26. Disponible en http://rcientificas.uninorte.edu.co/index.php/pensamiento/article/view/3977

Bateman, T. S. \& Snell, S. A. (2005). Administración: Un nuevo panorama competitivo. (4 ed.). México, D.F.: McGraw-Hill/Interamericana.

Caro, F. J. (2006). Gestión de Empresas Informativas. Madrid: McGraw Hill.

Cazallo, A., Barragán, C., Meñaca, I., Lechuga, J., Uribe, C. y Martínez, H. (2019). Mercosur y la Alianza del Pacífico. Dos modelos de competitividad - país. Espacios, 40(18), 26. Disponible en https://www.revistaespacios.com/ a19v40n18/19401826.html

Chumaceiro, A. y Hernández, J. (2012). La legislación tributaria venezolana como promotora de la responsabilidad social empresarial. Oikos, 16(33), 53-68. Disponible en http://ediciones.ucsh.cl/ojs/index.php/Oikos/article/view/1041

Chumaceiro, A., Chirinos, E. y Reyes, I. (2016). Antecedentes de políticas y organizaciones sociales orientadas a la promoción de la responsabilidad social empresarial en el siglo XX. Búsqueda, 3(16), 20-32. https://doi. org/10.21892/01239813.163

Chumaceiro, A., Hernández, J. y Velazco, J. (2015). Reflexiones sobre los modelos de políticas públicas de responsabilidad social empresarial en Europa. Oikos, 19(39), 77-91. Disponible en http://ediciones.ucsh.cl/ojs/index.php/Oikos/article/view/987

Cubillos, C., Montealegre, J.y Delgado, A. (2016). Responsabilidad social empresarial y Stakeholders en organizaciones de tamaños y actividades diferentes. Económicas CUC, 37(2), 115-136. https://doi.org/10.17981/econcuc.37.2.2016.06 
Durán, S. E., Crissien, J. E., Virviescas, J. y García, J. E. (2017). Estrategias gerenciales para la formación de equipos de trabajos en empresas constructoras del Caribe colombiano. Espacios, 38(13), 24. Disponible en https://www.revistaespacios.com/a17v38n13/17381324.html

Ferré, C. y Tolotti, C. (2010). Un modelo de análisis en Latinoamérica: La responsabilidad social corporativa (RSC) de las empresas de comunicación. Cuadernos de Información, (27), 97-110. https://doi.org/10.7764/cdi.27.26

García, J. E., Durán, S. E., Cardeño, E., Prieto, R., García, E. R. y Paz, A. (2017). Proceso de planificación estratégica: Etapas ejecutadas en pequeñas y medianas empresas para optimizar la competitividad. Revista Espacios, 38(52), 16. Disponible en https://www.revistaespacios.com/a17v38n52/a17v38n52p16.pdf

García, J., Cazallo, A., Barragan, C. E., Mercado, M., Olarte, L. y Meza, V. (2019). Indicadores de Eficacia y Eficiencia en la gestión de procura de materiales en empresas del sector construcción del Departamento del Atlántico, Colombia. Espacios, 40(22), 1-16. Disponible en http://www.revistaespacios.com/ a19v40n22/19402216.html

García, J., Durán, S., Parra, M. y Martínez, H. (2018). Inserción, integración y equidad en el ámbito laboral: Escenario empresarial posconflicto en Colombia. Revista de ciencias sociales, 24(3), 36-49. Disponible en https://produccioncientificaluz.org/index.php/rcs/article/view/24920

García, M., García, J. e Cabello, J. (2017). Eficiencia en el uso de los recursos y producción más limpia (recp) para la competitividad del sector hotelero. Revista de Gestão Social e Ambiental-RGSA, 11(2), 18-35. https://doi.org/10.24857/rgsa. v11i2.1252

González, M. (2005). El porqué de la responsabilidad social corporativa. Boletín Económico de Información Comercial Española, (2813), 45-58.

Guédez, V. (2006). Ética y práctica de la responsabilidad social empresarial. Caracas: Planeta.

Hartman, L. P. \& Werhane, P. H. (2013). Proposition: Shared value as an incomplete mental model. Business Ethics Journal Review, 1(6), 36-43. Available from https://businessethicsjournalreview.com/2013/02/26/hartman-on-porter/

Hernández, H., Muñoz, D. y Barrios, I. (2017). Estilos gerenciales y su influencia en la generación de valor de las Instituciones Prestadoras de Salud de la Región Caribe. Económicas CUC, 38(1), 133-146. https://doi.org/10.17981/econcuc.38.1.06

Hernández, R., Fernández, C. y Baptista, P. (2014). Metodología de la Investigación. México, D.F.: McGraw-Hill Interamericana.

Huerta, E. y García, J. (2009). Estrategias de gestión ambiental: Una perspectiva de las organizaciones modernas. Revista Clío América, 3(5), 15-30. Disponible en http://revistas.unimagdalena.edu.co/index.php/clioamerica/article/view/379

Klisberg, B. (2003). Responsabilidad Social para Latinoamericanos. Madrid: Deusto. Melamed-Varela, E., Blanco-Ariza, A. y Rodríguez-Calderón, G. (2018). Creación de valor compartido: estado y contribuciones a la sostenibilidad corporativa. Revista Escuela de Administración de Negocios, (85), 37-56. https://doi. org/10.21158/01208160.n85.2018.2047 
Méndez, C. (2005). La responsabilidad social de empresarios y de empresa. Caracas: Grupo Editorial Strategos Consultores.

Moliner, M. (2004). Marketing social la gestión de las causas sociales. Madrid: ESIC.

Olcese, A., Rodríguez, M. Á. y Alfaro, J. (2008). Manual de la Empresa Responsable y Sostenible. Conceptos y herramientas de la Responsabilidad Social Corporativa o de la Empresa. Madrid: McGraw-Hill.

Orozco, J. A. y Ferré, C. (2011). Los Stakeholders de las empresas de comunicación en el ámbito de la responsabilidad social corporativa. Folios, (25), 107-125. Disponible en https://revistas.udea.edu.co/index.php/folios/article/ view/10601

Paz, A., Harris, J. y Franco, F. (2016). Responsabilidad social gestión compartida con el emprendedor social en empresas mixtas del sector petrolero. Económicas CUC, 37(2), 47-68. https://doi.org/10.17981/econcuc.37.2.2016.03

Paz, A., Prieto, R. y García, J. (abril, 2015). Responsabilidad social como alianza para el desarrollo sostenible en empresas carboníferas. En, Cierg 2015, Primer Congreso Internacional de Energías Renovables, Riohacha, Guajira, Colombia.

Pelekais, C., Finol, M., Neuman, N., Carrasquero, E., García, J. y Leal, M. (2012). El $A B C$ de la investigación, un encuentro con la ciencia. Maracaibo: Astro Data.

Rabasa, B. (2005). Marketing Social. Madrid: Pirámide.

Sisiruca, M. y Salazar, C. (2014). Valores éticos de la responsabilidad social interna en centros de producción audiovisual. Económicas CUC, 35(1), 79-90. Recuperado de https://revistascientificas.cuc.edu.co/economicascuc/article/ view/259

Villasmil, M. y Crissien, T. (2015). Cambio de paradigma en la gestión universitaria basado en la teoría y praxis de la reingeniería. Económicas $C U C, 36(1)$, 126-142. Recuperado de https://revistascientificas.cuc.edu.co/economicascuc/ article/view/678

Weiss, W. J. (2006). Ética en los negocios: Un enfoque de administración de los Stakeholders y de casos. (4 ed.). México, D.F.: Thomson.

Yepes, G. A., Peña, W. y Sánchez, L. F. (2007). Responsabilidad Social Empresarial. Fundamentos y aplicación en las organizaciones de hoy. Bogotá, D.C.: Universidad Externado de Colombia.

BIODATA

Camilo Barragán Morales es Administrador de Empresas, especialista en Gerencia e Innovación y candidato a Magister en Administración de la Universidad Simón Bolívar (Barranquilla, Colombia). Miembro del grupo de investigación Pensamiento Contable y Gestión Internacional. https://orcid.org/0000-0002-81971727 
Jesús García-Guiliany es Ingeniero Industrial con Magister en Mercadeo. Doctor en Ciencias Gerenciales de la Universidad Rafael Belloso Chacin (Venezuela) e Investigador Senior Colciencias, miembro del grupo de investigación Gestión Organizacional. https://orcid.org/0000-0003-3777-3667

Víctor Meza-Rodríguez es Administrador de Empresas de la Universidad Simón Bolívar (Barranquilla, Colombia) y miembro del grupo de investigación Gestión Organizacional. https://orcid.org/0000-0003-4948-8257

Maria Mercado Zapata es Administradora de Empresas Universidad de la Costa (Colombia). https://orcid.org/0000-0001-7203-9068

Lucy Olarte Durán es profesional en Comercio y Negocios Internacionales de la Universidad Simón Bolívar (Barranquilla, Colombia). https://orcid.org/0000-0002$5562-4940$ 